\section{(1)}

CrossMark

\title{
Chronic blood exchange transfusions in the management of pre-capillary pulmonary hypertension complicating sickle cell disease
}

\author{
Matthieu Turpin ${ }^{1,2,3}$, Christelle Chantalat-Auger ${ }^{1,4,11}$, Florence Parent ${ }^{1,2,3,11}$, \\ Francoise Driss ${ }^{1,4}$, Francois Lionnet ${ }^{5}$, Anoosha Habibi ${ }^{6,7}$, Bernard Maître ${ }^{8}$,

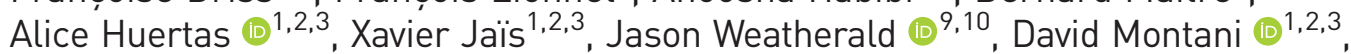 \\ Olivier Sitbon (10 ${ }^{1,2,3}$, Gérald Simonneau ${ }^{1,2,3}$. Fréderic Galactéros ${ }^{6,7}$ \\ Marc Humbert (10 ${ }^{1,2,3}$, Pablo Bartolucci $i^{6,7}$ and Laurent Savale (1) $1,2,3^{\prime}$
}

\begin{abstract}
Affiliations: 'Université Paris-Sud, Faculté de Médecine, Université Paris-Saclay, Le Kremlin-Bicêtre, France. ${ }^{2}$ AP-HP, Service de Pneumologie, Centre de Référence de l'Hypertension Pulmonaire Sévère, Hôpital Bicêtre, Le Kremlin-Bicêtre, France. ${ }^{3}$ INSERM UMR_S 999, Hôpital Marie Lannelongue, Le Plessis Robinson, France. ${ }^{4}$ AP-HP, Service de Médecine Interne, Hôpital Bicêtre, Le Kremlin-Bicêtre, France. ${ }^{5}$ AP-HP, Service de Médecine Interne, Hôpital Tenon, Paris, France. ${ }^{6}$ AP-HP, Unité des Maladies Génétiques du Globule Rouge, Centre de Référence des Pathologies du Globule Rouge, Hôpitaux Universitaire Henri Mondor, Créteil, France. ${ }^{7}$ UPEC, Institut Mondor de Recherche Biomédicale (IMRB), INSERM U955, DHU A-TVB, Créteil, France. ${ }^{8}$ AP-HP, Unité de Pneumologie, Hôpitaux Universitaires Henri Mondor, Créteil, France. ${ }^{9}$ Division of Respirology, Dept of Medicine, University of Calgary, Calgary, AB, Canada. ${ }^{10}$ Libin Cardiovascular Institute of Alberta, Calgary, AB, Canada. ${ }^{11}$ These two authors contributed equally to this work.
\end{abstract}

Correspondence: Laurent Savale, Université Paris-Sud, Centre de Référence de l'Hypertension Pulmonaire Sévère, Service de Pneumologie et Soins Intensifs Respiratoires, Hôpital Bicêtre, 78 Rue du Général Leclerc, 94270 Le Kremlin-Bicêtre, France. E-mail: laurent.savaledaphp.fr

@ERSpublications

Chronic exchange transfusions may be a potential therapeutic option in pre-capillary pulmonary hypertension complicating sickle cell disease http://ow.ly/BrMj30loRBY

Cite this article as: Turpin M, Chantalat-Auger C, Parent F, et al. Chronic blood exchange transfusions in the management of pre-capillary pulmonary hypertension complicating sickle cell disease. Eur Respir J 2018; 52: 1800272 [https://doi.org/10.1183/13993003.00272-2018].

ABSTRACT The long-term effects of chronic blood exchange transfusions (BETs) on pre-capillary pulmonary hypertension complicating sickle cell disease (SCD) are unknown.

13 homozygous SS SCD patients suffering from pre-capillary pulmonary hypertension and treated by chronic BETs were evaluated retrospectively. Assessments included haemodynamics, New York Heart Association Functional Class (NYHA FC), 6-min walk distance (6MWD) and blood tests.

Before initiating BETs, all patients were NYHA FC III or IV, median (range) 6MWD was $223(0-501) \mathrm{m}$ and median (range) pulmonary vascular resistance (PVR) was 3.7 (2-12.5) Wood Units. After a median number of 4 BET sessions, all patients had improved to NYHA FC II or III. Significant improvements in haemodynamics were observed, including a decrease in PVR $(\mathrm{p}=0.01)$. There was a trend to higher $6 \mathrm{MWD}(\mathrm{p}=0.09)$. Median (range) follow-up time after initiation of BETs was 25 (6-53) months. During this period, two patients decided to stop BETs. One of them died from acute right heart failure and the other experienced worsening pulmonary hypertension. Two other patients died during follow-up at 25 and 54 months after BET initiation.

Chronic BETs may be a potential therapeutic option in pre-capillary pulmonary hypertension complicating SCD, leading to significant clinical and haemodynamic improvements. These data must be confirmed in a prospective study. 


\section{Introduction}

Chronic pre-capillary pulmonary hypertension has been identified as a devastating complication of sickle cell disease (SCD). Haemodynamic studies from large cohorts of SCD patients estimated a prevalence of pre-capillary pulmonary hypertension between $2.8 \%$ and $3.75 \%[1,2]$. The main histological lesion seen in SCD patients with pre-capillary pulmonary hypertension is pulmonary vascular remodelling combined with distal vascular occlusion from in situ thrombosis, which leads to progressive elevation of pulmonary vascular resistance (PVR). The pathophysiological mechanisms of pre-capillary pulmonary hypertension in this setting are not yet fully understood, but multiple potential mechanisms contribute [3]. Chronic haemolysis may lead to depletion of nitric oxide (NO) and its precursors, thereby impairing vascular endothelial function [4-6]. A hypercoagulable state in SCD is promoted by several pathways, including NO depletion, platelet activation and functional asplenia, which could lead to in situ thrombosis [7-9]. A high cardiac output due to chronic anaemia may also induce endothelial dysfunction from flow-induced shear stress. Whatever the underlying mechanism, it has been clearly established that pre-capillary pulmonary hypertension has a major impact on the functional status of patients with SCD. Severe dyspnoea and 6-min walk distance (6MWD) impairment due to pulmonary hypertension have been observed in all clinical studies. In addition, the prognosis of SCD patients with pre-capillary pulmonary hypertension appears to be severely compromised $[1,10,11]$.

Owing to the detrimental impact of pulmonary hypertension on functional status and overall survival, early detection and adapted management of pulmonary hypertension in this population are necessary. Two main treatment strategies have been proposed: 1) the use of pulmonary arterial hypertension (PAH)-targeted therapies and 2) the optimisation of SCD treatment using hydroxyurea and/or chronic transfusion therapy [12]. However, specific practice guidelines on the management of SCD-associated pre-capillary pulmonary hypertension remain difficult to establish due to a paucity of evidence. Specific clinical trials are lacking or have failed to demonstrate a positive effect of PAH-targeted therapies in this very specific setting $[13,14]$. Moreover, no specific trials have been performed to evaluate the effect of hydroxyurea on pre-capillary pulmonary hypertension.

Chronic blood exchange transfusions (BETs) are an interesting therapeutic option which might decrease the proportion of sickle haemoglobin $(\% \mathrm{HbS})$, optimise the haemoglobin level and limit the effect of chronic haemolysis on pulmonary endothelial function. The beneficial effect of this therapeutic approach on morbidity and mortality of SCD patients with other severe complications has already been reported [15]. A brief communication observed a beneficial effect of this therapeutic approach in two SCD patients with pulmonary hypertension, but invasive haemodynamic data were lacking [16]. Considering the prognostic impact of pre-capillary pulmonary hypertension on SCD patients, we proposed chronic BETs, most often by erythrocytapheresis (ECP), in the management of patients referred to the French Pulmonary Hypertension Reference Centre (Le Kremlin-Bicêtre, France). The aim of the current study was to show the biological, clinical and haemodynamic effects of chronic BETs in these patients.

\section{Methods}

\section{Design}

This was a retrospective study that aimed to describe the short- and long-term outcomes of all homozygous SS SCD patients with pre-capillary pulmonary hypertension who were treated with chronic BETs in the French Pulmonary Hypertension Reference Centre between October 2012 and March 2017. The observational period ended in September 2017, 6 months after treatment initiation of the last patient. Characteristics of these patients at baseline were compared with SS SCD patients with pre-capillary pulmonary hypertension followed during the same period but not treated with BETs.

This retrospective study complied with the Declaration of Helsinki. Although French law does not require ethics committee approval or informed consent for retrospective data collection, the data were anonymised and compiled according to the requirements of the Commission Nationale Informatique et Liberté, the organisation dedicated to privacy, information technology and civil rights in France. The committee approved the methods used to collect and analyse data on May 24, 2003 (approval 842063).

\section{Patients}

All patients were homozygous for HbS. Pre-capillary pulmonary hypertension was screened by transthoracic echocardiography and systematically confirmed by right heart catheterisation (RHC), showing a mean pulmonary arterial pressure of $\geqslant 25 \mathrm{mmHg}$ and a pulmonary capillary wedge pressure of $\leqslant 15 \mathrm{mmHg}$ [17]. Cardiac output was measured by thermodilution. Haemodynamic assessment was systematically performed at least 1 month after an acute vaso-occlusive crisis. 
All patients whose diagnosis of pulmonary hypertension was confirmed by RHC underwent extensive investigations in order to identify additional causes or risk factors of PAH (congenital heart disease, portal hypertension, HIV infection and connective tissue disease). In addition, the presence of chronic lung disease and chronic thromboembolic pulmonary hypertension (CTEPH) was systematically assessed by performing pulmonary function tests, high-resolution computed tomography (CT) of the chest and ventilation/perfusion lung scintigraphy followed by CT pulmonary angiography if needed. Patients with CTEPH necessitating management with pulmonary angioplasty and/or endarterectomy were excluded.

\section{Treatment regimen}

All patients were treated with BET as first-line therapy at the time of pulmonary hypertension diagnosis or after a clinical and haemodynamic worsening despite the use of PAH therapies. The decision to initiate an exchange transfusion programme was based on a multidisciplinary evaluation of the severity of SCD and pulmonary hypertension assessed by clinical, functional and haemodynamic parameters, but also on the ability to establish adequate venous access. Immunisation history and previous known delayed haemolytic transfusion reaction (DHTR) episodes were recorded. Patients with a history of DHTRs were not included in the transfusion programme. The transfusion protocols used were those recommended in France. All patients were transfused with leukoreduced red blood cell (RBC) units and taking into account the potential risk of immunisation. Nonimmunised patients received prophylactic $R h(D, C, E, c$ and $e)-$ and K-matched RBCs. Patients known to have a clinically significant antibody other than antibodies against $\mathrm{Rh}$ and $\mathrm{K}$ (e.g. antibodies against FY, JK, MNS and DO) received extended-match RBCs for the antibodies and, if appropriate resources were available, extended-matched for FY, JK and MNS. RBCs were at least matched for Rh (D, C, E, c and e) and KEL antigens. Patient plasma was always cross-matched before transfusion with samples from RBC units [18].

ECP was preferred over manual exchange in most cases. Patients in ECP programmes had a session every 5-6 weeks and received RBCs at $20-25 \mathrm{~mL} \cdot \mathrm{kg}^{-1}$ body weight on each session, as described previously [19]. All patients on ECP were transfused using an apheresis machine (Com-Tec; Fresenius Kabi, Paris, France) with a continuous flow system under isovolaemic conditions. A post-procedure haematocrit of $\geqslant 30 \%$ and a post-procedure $\mathrm{HbS}$ level of $<30 \%$ were targeted. The procedures were performed in the transfusion unit or in the intensive care unit in cases with severe right ventricular dysfunction.

The off-label use of PAH therapies, i.e. endothelin receptor antagonists (ERAs), phosphodiesterase type 5 (PDE5) inhibitors or prostanoids, was decided after a multidisciplinary discussion of the expected benefits and risks of this medical strategy.

All patients received supportive therapies in accordance with pulmonary hypertension guidelines, i.e. diuretics to control signs and symptoms of right ventricular failure (including peripheral oedema) and long-term oxygen therapy if hypoxaemia was present. Initiation of long-term anticoagulation therapy was left to the discretion of physicians according to the assessment of the possible contribution from thrombosis to pulmonary hypertension (according to ventilation/perfusion lung scintigraphy followed by angio-CT, if needed) and the risk of bleeding.

\section{Patient assessments}

All patients underwent a complete evaluation at the time of pulmonary hypertension diagnosis and before starting BET therapy, including assessment of modified New York Heart Association Functional Class (NYHA FC), physical examination, routine blood tests, nonencouraged 6-min walk distance (6MWD) and resting haemodynamic variables measured by RHC. Patients were reassessed for all parameters at shortand long-term time intervals. Haemodynamic assessment was repeated 3-6 months after BET initiation, then every year or in cases of clinical worsening. Clinical, functional, biological and haemodynamic assessments during follow-up were systematically performed immediately prior to the next BET. Noninvasive assessments were also repeated every 4-6 months. The first and last evaluations while on BET therapy were analysed.

\section{Statistical analysis}

Results are expressed as median (range). Statistical significance between patients treated and not treated with BETs was tested using the nonparametric Mann-Whitney U-test. Baseline and post-baseline values for 6MWD and haemodynamic variables were compared using a Wilcoxon matched-pairs signed-rank test. Changes in NYHA FC were compared using the Chi-squared test. A p-value $<0.05$ was considered statistically significant. 


\section{Results}

During the study period, a total of 38 patients with SCD and associated pre-capillary pulmonary hypertension were managed by the French Pulmonary Hypertension Reference Centre (figure 1). 11 of them were excluded because of having SC SCD. One other patient with SS SCD was excluded because of a diagnosis of proximal CTEPH with an indication for surgical endarterectomy. Finally, 26 SS SCD patients with pre-capillary pulmonary hypertension were included and analysed. 13 were treated with chronic BETs. The characteristics of these 13 patients at the time of pulmonary hypertension diagnosis are shown in table 1 and compared with the 13 patients who were not treated with chronic BETs. Seven of the patients were female and the median (range) age was 43 (27-57) years. Median PVR at the time of pulmonary hypertension diagnosis was 2.7 Wood Units (WU). Seven patients had PVR $>3$ WU. Six patients were unable to perform a 6MWD test at baseline because of severe dyspnoea and were NYHA FC IV. Patients not treated with BETs trended towards having less severe haemodynamics at baseline and 54\% of them were in NYHA FC I or II at baseline. One of them had a history of alloimmunisation and DHTRs that prohibited transfusion.

\section{Treatment history}

In the group of patients treated with BETs, hydroxyurea had been initiated before the diagnosis of pulmonary hypertension in 11 patients and was continued in all cases after pulmonary hypertension diagnosis. The proportion of patients treated by hydroxyurea was significantly lower in the control group ( $43 \%$ versus $85 \%$; $\mathrm{p}=0.02$ ).

Before chronic BET programme initiation, six patients had a history of RBC antibodies, which were found just before the first session in one case. All of these antibodies were systematically taken into account in the long-term management. Chronic BETs were initiated as first-line therapy after pulmonary hypertension diagnosis in 10 patients. For these patients, evaluation before BET initiation corresponds to the baseline evaluation. Targeted PAH therapy with bosentan was simultaneously introduced in two patients (patients 10 and 12). In three other patients, chronic BETs were initiated 2, 28 and 62 months after pulmonary hypertension diagnosis, respectively (two of them had previously been treated with PAH therapies). Chronic BETs were added in these cases because of clinical, functional and haemodynamic worsening despite the use of PAH therapies. The characteristics of all patients before the initiation of BETs are described in table 2. Median (range) PVR was 3.7 (2-12.5) WU and all patients were in NYHA FC III or IV. BETs were performed manually in three patients (patients 2, 3 and 4) and by ECP in the remaining 10 patients. Anticoagulation therapy was initiated in six patients after pulmonary hypertension diagnosis due to heterogeneous perfusion on ventilation/perfusion lung scintigraphy without segmental defects.

\section{Short-term efficacy}

Re-evaluations were systematically performed after a median (range) number of 4 (1-6) BET procedures. Short-term effects on NYHA FC, 6MWD and haemodynamics that were assessed right before the next $\mathrm{BET}$ are reported in table 2. Median (range) \%HbS measured immediately prior to BET at the time of haemodynamic assessment was $43 \%$ (14-59\%). All patients improved to NYHA FC II or III. After initiation of BETs, 10 patients (77\%) were in NYHA FC I or II. There was a $175 \mathrm{~m}$ improvement in

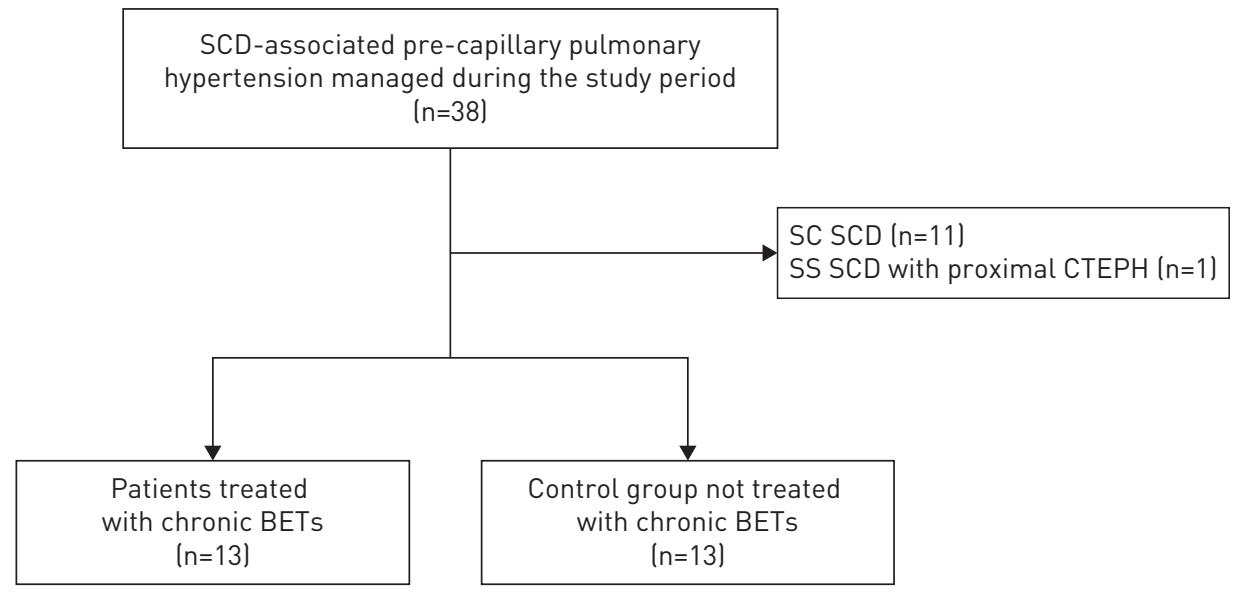

FIGURE 1 Flowchart of sickle cell disease (SCD)-associated pre-capillary pulmonary hypertension patients managed by the French Pulmonary Hypertension Reference Centre during the study period. SC: heterozygous; SS: homozygous; CTEPH: chronic thromboembolic pulmonary hypertension; BET: blood exchange transfusion. 
TABLE 1 Characteristics of sickle cell disease (SCD) patients at time of diagnosis of pre-capillary pulmonary hypertension

Group treated with BETs

Group not treated with BETs

p-value

Patients $\mathbf{n}$

Age years

Female

History of vaso-occlusive disease

$>2$ vaso-occlusive crises per year

History of acute chest syndrome

History of leg ulcers

History of acute thromboembolic disease

Hydroxyurea therapy

History of splenectomy

Laboratory values

Haemoglobin $\mathrm{g} \cdot \mathrm{dL}^{-1}$

Lactate dehydrogenase IU. $\mathrm{L}^{-1}$

Total bilirubin $\mu \mathrm{mol} \cdot \mathrm{L}^{-1}$

Creatinine $\mu \mathrm{mol} \cdot \mathrm{L}^{-1}$

Pulmonary function

FEV $1 \%$ pred

FVC \% pred

TLC \% pred

DLco \% pred

$\mathrm{PaO}_{2} \mathrm{mmHg}$

$\mathrm{PaCO}_{2} \mathrm{mmHg}$

NYHA FC

I/II

III/IV

6MWD m

Tricuspid regurgitant velocity $\mathrm{m} \cdot \mathrm{s}^{-1}$

Haemodynamics

Right atrial pressure $\mathrm{mmHg}$

Mean pulmonary artery pressure $\mathrm{mmHg}$

Pulmonary capillary wedge pressure $\mathrm{mmHg}$

Cardiac index L. $\mathrm{min}^{-1} \cdot \mathrm{m}^{-2}$

Pulmonary vascular resistance WU

$\mathrm{SvO}_{2} \%$
13

$43(27-57)$

7 (54)

$11(85)$

7 (54)

11 (85)

$4(31)$

4 (31)

$11(85)$

1 (8)

$8.3(7-9.6)$

$937(415-3034)$

$35(22-92)$

84 (36-137)

$65(29-76)$

$65(29-81)$

$69(36-95)$

$37(25-68)$

$68(43-100)$

$37(27-46)$

2 (15)

$11(85)$

$234(0-501)$

$3.95(3.01-4.8)$

$9(7-22)$

$36(30-48)$

$10(4-14)$

$5.5(2.4-7.5)$

$2.7(1.9-9.3)$

$60(32-80)$
13

$48(29-66) \quad 0.5$

$10(77)$

$13(100)$

9 (69)

9 (69)

4 (31)

2 (15)

6 (43)

2 (15)

0.2

0.1

0.4

0.3

1

0.3

0.02

0.5

$8.5(6.6-11.5)$

785 (678-1464)

$24(11-41)$

71 (44-89)

0.6

0.8

0.06

0.2

61 (50-95)

0.9

67 (50-97)

71 (51-99)

44 (29-78)

75 (64-83)

38 (30-43)

\section{7}

0.2

0.8

0.6

0.8

7 (54)

$6(46)$

395 (210-527)

$3.16(2.50-4.05)$

0.04

0.06

0.026

$3(2-8)$

30 (25-44)

0.001

$10(5-14)$

$4.9(3.2-6.6)$

0.02

$2.7(1.4-4)$

67 (57-77)

Data are presented as $\mathrm{n}$, median (range) or $\mathrm{n}(\%)$, unless otherwise stated. BET: blood exchange transfusion; FEV1: forced expiratory volume in $1 \mathrm{~s}$; FVC: forced vital capacity; TLC: total lung capacity; DLCo: diffusing capacity of the lung for carbon monoxide; $P$ aO ${ }_{2}$ : arterial oxygen tension; $\mathrm{PaCO}_{2}$ : arterial carbon dioxide tension; NYHA FC: New York Heart Association Functional Class; 6MWD: 6-min walk distance; WU: Wood Units; $\mathrm{SvO}_{2}$ : mixed venous oxygen saturation.

6MWD $(p=0.09)$. A significant decrease in tricuspid regurgitant velocity was observed during the follow-up ( $p=0.0002$ ). Pulmonary haemodynamics improved with a decrease in PVR by a median of $24 \%$ $(\mathrm{p}=0.01)$. Individual PVR changes are illustrated in figure 2. The decrease in PVR exceeded $25 \%$ in seven out of 13 patients. Median \%HbS at time of RHC was similar between this subgroup and the others $(43 \%$ versus $42.5 \%$, respectively). All of them were treated with ECP. In contrast, no significant change in PVR was observed in the three patients manually exchanged (patients 2, 3 and 6). In two patients, when bosentan was simultaneously initiated with ECP, the PVR decreased from 5.8 to $3.28 \mathrm{WU}$ (patient 10) and from 6.03 to $3.48 \mathrm{WU}$ (patient 12).

Serum levels of lactate dehydrogenase (LDH) significantly decreased after BET initiation. No correlation was found between percentage decrease in LDH and percentage change in PVR.

\section{Long-term evolution}

The median (interquartile range (IQR)) follow-up time of patients treated with BETs was 27 (17-45) months. Seven patients were assessed at least three times while receiving chronic BETs. The last evaluation of these patients was conducted at median (range) 25 (6-53) months after BET initiation. The remaining six patients did not undergo long-term evaluation for the following reasons: four had only one evaluation at cut-off, one patient refused to perform a repeat invasive haemodynamic evaluation and one decided to stop BET before the third evaluation. Among patients who were evaluated at least three times, 
TABLE 2 Short-term efficacy of chronic blood exchange transfusions (BETs)

Last evaluation before BETs

After a median of 4 BETs

p-value

\begin{tabular}{|c|c|c|c|}
\hline \multicolumn{4}{|l|}{ NYHA FC } \\
\hline $\mathrm{I} / \mathrm{II}$ & $0(0)$ & $10(77)$ & \multirow[t]{2}{*}{$<0.001$} \\
\hline IV & 5 (39) & $0(0)$ & \\
\hline \multicolumn{4}{|l|}{ Haemodynamics } \\
\hline Right atrial pressure $\mathrm{mmHg}$ & $9(4-22)$ & $5(0-17)$ & 0.04 \\
\hline Mean pulmonary arterial pressure $\mathrm{mmHg}$ & $40(31-58)$ & $35(22-48)$ & 0.005 \\
\hline Pulmonary vascular resistance WU & $3.7(2.0-12.5)$ & $2.8(1.59-8.30)$ & 0.01 \\
\hline $\mathrm{SvO}_{2} \%$ & $58(32-75)$ & $66(42-77)$ & 0.14 \\
\hline Tricuspid regurgitant velocity $\mathrm{m} \cdot \mathrm{s}^{-1^{\#}}$ & $4.06(3.01-5.27)$ & $3.83(2.34-4.87)$ & 0.0002 \\
\hline \multicolumn{4}{|l|}{ Laboratory values } \\
\hline Haemoglobin $\mathrm{g} \cdot \mathrm{dL}^{-1}$ & $8.0(7.0-9.5)$ & $9.3(7.7-12.9)$ & 0.15 \\
\hline Lactate dehydrogenase IU. $\mathrm{L}^{-1}$ & $1014(415-2691)$ & 775 (419-1858) & 0.001 \\
\hline
\end{tabular}

Data are presented as $\mathrm{n}(\%)$ or median (range), unless otherwise stated. NYHA FC: New York Heart Association Functional Class; 6MWD: 6-min walk distance; WU: Wood Units; $\mathrm{SvO}_{2}$ : mixed venous oxygen saturation; BNP: brain natriuretic peptide; NT-proBNP: N-terminal pro-BNP; $\% \mathrm{HbS}$ : proportion of sickle haemoglobin. ${ }^{\#}$ : tricuspid regurgitant velocity measurement was not systematically performed right before BETs.

improvements in haemodynamic variables observed in the short term were largely maintained, with significant improvement in NYHA FC and increases in cardiac index and PVR over baseline (figure 3). During the follow-up period, only one patient developed new RBC antibodies (MNS4), 3 years after inclusion in the programme. In contrast, no patient developed a DHTR during the study period. Moreover, the level of ferritinaemia did not change during the follow-up period (median (range) 362 (17-2525) versus 469.5 (20-2128) $\mu \mathrm{g} \cdot \mathrm{L}^{-1}$; nonsignificant).

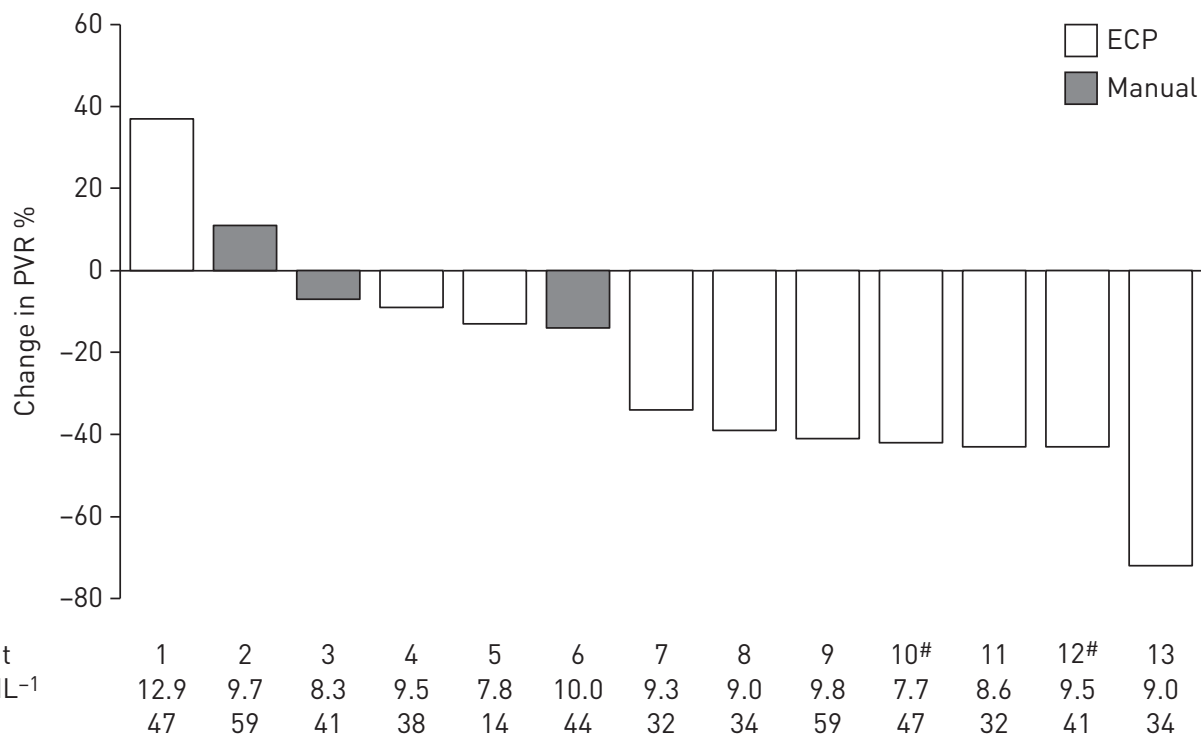

FIGURE 2 Change in pulmonary vascular resistance (PVR) after blood exchange transfusions (BETs) (individual data). ECP: erythrocytapheresis; $\mathrm{Hb}$ : haemoglobin; \% $\mathrm{HbS}$ : proportion of sickle $\mathrm{Hb}$. Median (range) number of exchange sessions at time of haemodynamic assessment was 4 (1-6). Manual exchanges were performed in patients 2, 3 and 6, and ECP in others. \#: bosentan $(62.5 \mathrm{mg}$ twice daily) was initiated at the same time as the BET programme in patients 10 and 12 . 

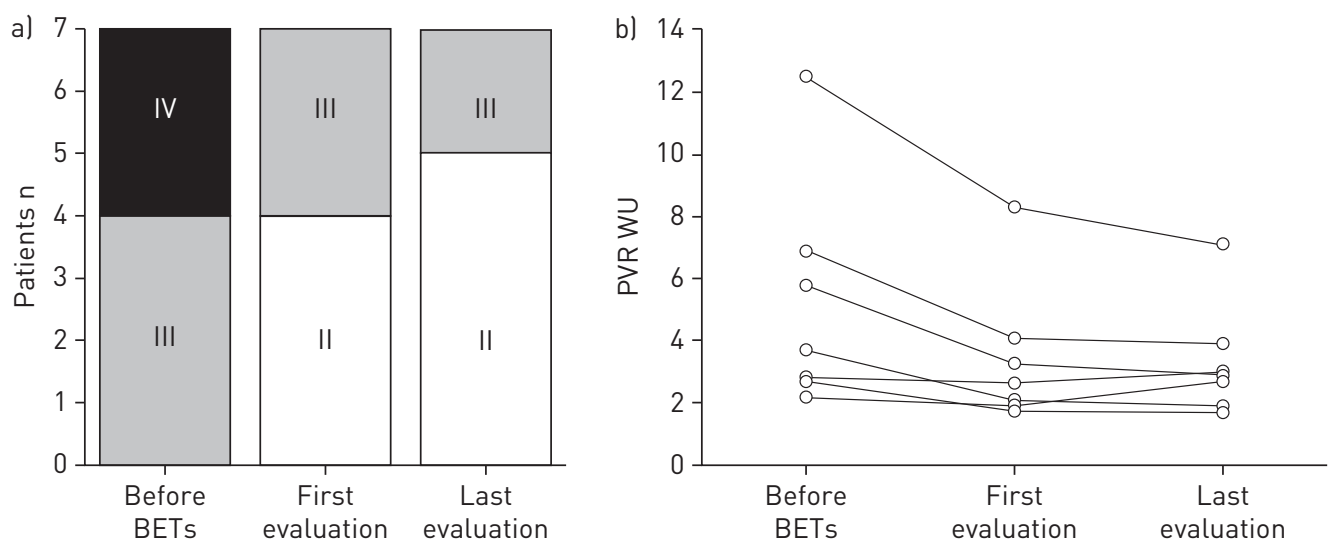

FIGURE 3 Long-term effects of chronic blood exchange transfusions (BETs) on a) New York Heart Association Functional Class (classes II-IV) and b) pulmonary vascular resistance (PVR) in seven patients evaluated at least three times. WU: Wood Units.

Two patients decided to stop BETs after 12 and 24 months (patients 2 and 11, respectively). Patient 11 was re-evaluated 6 months after exchange transfusion withdrawal. A subsequent clinical, function and haemodynamic assessment demonstrated a pulmonary hypertension worsening to NYHA FC class IV with a PVR increase from 1.9 to $4.59 \mathrm{WU}$ (figure 4). The other patient died from acute right heart failure 1 year after BET withdrawal.

Two other patients died from right heart failure during follow-up, at 25 and 54 months after BET initiation (patients 9 and 7, respectively). In these two patients, BETs had been initiated after PAH therapy failure in the context of severe pre-capillary pulmonary hypertension. Patient 9 developed sudden pulmonary hypertension worsening followed by acute right heart decompensation after the creation of an arteriovenous fistula for BETs.

The median (IQR) follow-up time of the control group was 53 (35-103) months. During the study period six of them died, including five of right heart failure.

\section{Discussion}

In this study, we describe a new potential treatment strategy in a series of 13 SCD patients with pre-capillary pulmonary hypertension and severe functional impairment. Patients who were managed

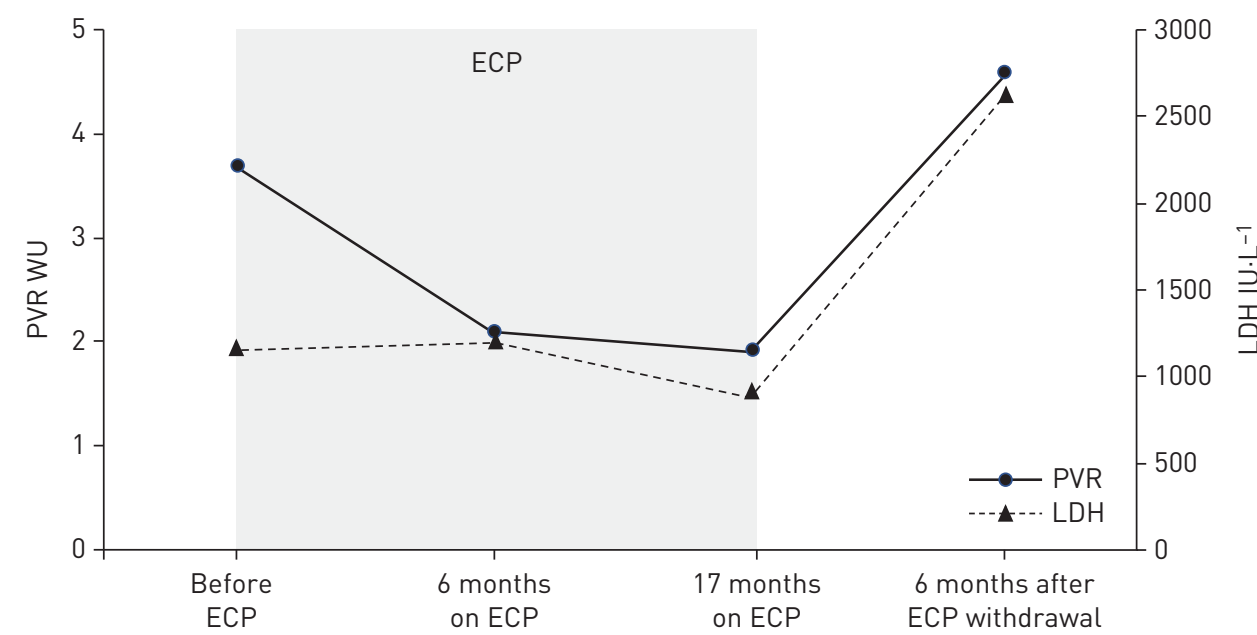

$\begin{array}{lcccc}\text { NYHA FC } & \text { IV } & \text { III } & \text { II } & \text { IV } \\ \mathrm{Hb} \mathrm{g} \cdot \mathrm{dL}^{-1} & 11.9 & 8.8 & 7.7 & 8.8 \\ \% \mathrm{HbS} & 79 & 32 & 40 & 82\end{array}$

FIGURE 4 Evolution of patient 11 after erythrocytapheresis (ECP) withdrawal. PVR: pulmonary vascular resistance; WU: Wood Units; LDH: lactate dehydrogenase; NYHA FC: New York Heart Association Functional Class; $\mathrm{Hb}$ : haemoglobin; \% $\mathrm{HbS}$ : proportion of sickle $\mathrm{Hb}$. 
with chronic BETs experienced improvements in NYHA FC and significant decreases in PVR. Furthermore, this effect seemed to persist over time in most patients. The cessation of chronic BETs in two patients led to death, due to right heart failure in one and to severe pulmonary hypertension worsening in the other.

All patients were assessed by RHC before and after the initiation of BETs. Clinical and haemodynamic profiles at baseline were in keeping with what has been previously observed in this particular population. Haemodynamics were characterised by moderate to severe elevation of mean pulmonary arterial pressure most often associated with high cardiac output. Despite the hyperdynamic status, half of our patients had PVR >3 WU. Pre-capillary pulmonary hypertension was associated with severe impairment of NYHA FC and exercise capacity at baseline in all patients. Serum LDH was also elevated, as described previously [1].

This is the first report of the long-term effects of chronic BETs in pre-capillary pulmonary hypertension with invasive haemodynamic evaluations throughout the follow-up process. In a previous communication, TsiTsikas et al. [16] described the short-term evolution of two SCD patients with pulmonary hypertension after automated BET. Pulmonary hypertension had been previously assessed by RHC, but follow-up invasive haemodynamic evaluations were not performed before and after transfusion. However, clinical evolution reported in these two clinical cases was very interesting, with a rapid and marked improvement of dyspnoea associated with resolution of hypoxia and a trend towards improvement of tricuspid regurgitation velocity and brain natriuretic peptide levels [16].

Our case series provides new evidence to support the efficacy of chronic BETs in the management of SCD patients with pre-capillary pulmonary hypertension. The short- and long-term follow-up analyses confirm the beneficial and substantial effect of exchanges on the functional capacities of patients. Another strength of our report is the systematic haemodynamic assessment confirming a marked improvement with a significant decrease in PVR $>25 \%$ in more than half of the patients. The unfavourable outcomes observed in patients who stopped the transfusion programme reinforce the likely beneficial effect of this treatment. In contrast, no death was observed in patients who underwent an exchange transfusion programme as first-line therapy at the time of pulmonary hypertension diagnosis and without interruption in the treatment.

An American Thoracic Society (ATS) clinical practice guideline suggested chronic transfusion in the management of pre-capillary pulmonary hypertension in SCD patients who have an increased risk for mortality (i.e. pulmonary hypertension confirmed by RHC) and who are not responsive to or are not candidates for hydroxyurea [12]. The authors of this ATS guideline rightly indicated that estimated effects of chronic transfusion were based on low-quality evidence [12]. Our report provides additional arguments supporting the use of this therapeutic approach in the management of severe patients. Of note, two randomised clinical trials reported beneficial effects of chronic transfusion therapy on cerebral vasculopathy associated with SCD, reducing the stroke rate in children with a transcranial Doppler velocity $>200 \mathrm{~cm} \cdot \mathrm{s}^{-1}$ in the middle cerebral or internal carotid artery [20, 21]. Elevated cerebral blood flow velocities in SCD are associated with severe anaemia, vessel stenosis and cerebral vasodilatation caused by tissue hypoxia. The authors of this Stroke Prevention Trial in Sickle Cell Anemia study suggested that chronic transfusion could reduce cerebral blood flow velocities and prevent stroke risk by correcting at least partially these abnormalities [20,21]. Similarly, we can postulate that BETs might partially reverse the pathophysiological mechanisms leading to PVR elevation in SCD.

It has been suggested that chronic haemolysis plays an important role in the pathophysiology of SCD-associated pulmonary hypertension. This hypothesis is based on biological and clinical arguments. Patients with pulmonary hypertension have a high rate of abnormal biological markers of chronic haemolysis [1, 11]. Scavenging of $\mathrm{NO}$ and its substrates by free haemoglobin could promote vasoconstriction, endothelial dysfunction and pulmonary vascular remodelling [4-6]. Chronic BETs reduce the HbS level and thus the impact of chronic haemolysis, as was suggested by the significant reduction in $\mathrm{LDH}$ observed in our patients after exchange.

However, chronic haemolysis is probably not the only physiological explanation of PVR elevation in SCD. Indeed, we did not find any correlation between $\% \mathrm{HbS}$ or percentage decrease in $\mathrm{LDH}$ and decrease in PVR after BET programme initiation. In situ thrombosis has been reported as an important pathological component of microvascular obstruction in previous studies [10]. Moreover, ventilation/perfusion lung scintigraphy highlighted peripheral defects in a high proportion of patients. Some cases of CTEPH have been described in patients with SS SCD and more frequently in patients with SC SCD [22, 23]. We could suggest that BETs might decrease the hypercoagulable state associated with SCD. As reported by TsiTsIKAS et al. [16], we also observed a rapid improvement of symptoms immediately after BETs. This suggests the possibility of an acutely reversible component of pre-capillary pulmonary hypertension, on which BETs could be effective. Prospective studies are mandatory to study the acute physiological effects of BETs on 
pulmonary haemodynamics, which could contribute to a better understanding of the physiological mechanism of pre-capillary pulmonary hypertension complicating SCD. Changes in blood viscosity induced by BETs also need to be studied. We have also observed that manual exchanges were less beneficial to patients, compared with ECP. Indeed, haematocrit control is much more difficult with manual exchanges, which are associated with a higher risk of blood hyperviscosity. This could contribute to the disappointing effects of manual exchanges on haemodynamics observed in the present report. Interestingly, a significant decrease of tricuspid regurgitant velocity was observed after a curative approach with myeloablative allogeneic haematopoietic stem cell transplantation. However, invasive haemodynamic assessment was lacking in this study [24]. More recently, a first case of pulmonary hypertension resolution assessed by RHC has been reported after successful haploidentical peripheral blood stem cell transplantation [25].

The potentially undesirable consequences of chronic BETs must be taken into account in order to appreciate the value of this medical approach on long-term outcomes of pre-capillary pulmonary hypertension complicating SCD. None of our 13 patients had DHTRs. The risk of iron overload must also be considered and regularly assessed in patients managed with chronic transfusions. However, the preferential use of ECP helps prevent iron accumulation. Good vascular access is required for automated exchanges, which can be a major difficulty in some patients with SCD and might not be a feasible option in all socioeconomics contexts. In most of our patients, the introduction of a central venous catheter was performed for each transfusion session. Despite this invasive approach, we did not observe any acute thromboembolic or infectious complications. An arteriovenous fistula had to be created in one patient (patient 9) due to difficult venous access. This procedure led to a sudden pulmonary hypertension worsening followed by acute right heart decompensation and death. This case has been previously reported by DeLville et al. [26].

Due to the foreseeable difficulties in maintaining a chronic BET approach over the very long term, other alternatives must be discussed and evaluated. There is no question that pre-capillary pulmonary hypertension complicating SCD requires active management, taking into account the critical impact of pulmonary hypertension on prognosis. Hydroxyurea has been shown to be effective in reducing the number of acute vaso-occlusive crises, episodes of acute chest syndrome, hospitalisations, transfusion use and probably mortality $[20,21]$. The beneficial effect on the prevention and progression of chronic complications needs to be properly evaluated. Hydroxyurea has been shown to reduce both the formation of $\mathrm{HbS}$ and haemolysis. These demonstrable effects suggest that this medical therapy could also be interesting in the management of pre-capillary pulmonary hypertension in SCD, but its effect on pulmonary haemodynamics is unknown [27]. In our study, the majority of patients were already treated by hydroxyurea at the time of pulmonary hypertension diagnosis in the group treated with BETs.

As mentioned in the Introduction, randomised clinical trials failed to demonstrate a positive effect of PAH-targeted therapies (ERAs or PDE5 inhibitors) in pulmonary hypertension complicating SCD, but these studies were not completed due to either a lack of enrolment in the ERA trial or an increased incidence of hospitalisation for pain in the PDE5 inhibitor trial $[13,14]$. In accordance, a relatively low proportion of our patients was treated with PAH therapies. The two patients who received bosentan at the same time as BET initiation presented with severe pre-capillary pulmonary hypertension at the time of diagnosis.

The main limitations of this report relate to its retrospective nature and the small number of patients. Accordingly, these data should be interpreted with caution. The impact of missing data was limited by the requirement that the data should be available before and after exchange therapy in the French Pulmonary Hypertension Registry. This report reflects real-life management. As mentioned earlier, the use of other therapies, e.g. PAH therapies or hydroxyurea in some patients, could have had a positive or negative impact on patient outcomes. However, the improvement observed in some patients exclusively treated with BETs as first-line therapy and the effect of exchange withdrawal on pulmonary hypertension evolution are important findings that should justify further studies.

In conclusion, we provide preliminary evidence supporting the short- and long-term benefits of chronic BETs, especially by ECP, in patients with pre-capillary pulmonary hypertension complicating SCD. Prospective clinical trials are needed to confirm the positive effect of chronic BETs on pre-capillary pulmonary hypertension complicating SCD.

Conflict of interest: M. Turpin has nothing to disclose. C. Chantalat-Auger has nothing to disclose. F. Parent has nothing to disclose. F. Driss has nothing to disclose. F. Lionnet has nothing to disclose. A. Habibi has nothing to disclose. B. Maître has nothing to disclose. A. Huertas has nothing to disclose. X. Jaïs reports grants and personal fees from Actelion and GSK, personal fees from MSD, grants from Bayer, outside the submitted work. J. Weatherald reports personal fees and nonfinancial support from Actelion and Bayer, personal fees from Novartis, and grants from the 
Canadian Vascular Network, outside the submitted work. D. Montani reports grants and personal fees from Actelion and Bayer, personal fees from BMS, GSK, MSD and Pfizer, outside the submitted work. O. Sitbon reports grants, personal fees and nonfinancial support from Actelion, Bayer, Merck and GSK, personal fees from Acceleron and Arena, outside the submitted work. G. Simonneau reports grants, personal fees and nonfinancial support from Actelion, Bayer, Merck and GlaxoSmithKline, personal fees from Acceleron and Arena, outside the submitted work. F. Galactéros has nothing to disclose. M. Humbert has relationships with drug companies including Actelion, Bayer, GSK, Novartis, Pfizer and United Therapeutics. In addition to being investigator in trials involving these companies, relationships include consultancy service and membership of scientific advisory boards. P. Bartolucci has nothing to disclose. L. Savale reports grants and personal fees from Actelion and Bayer, personal fees from MSD and GSK, during the conduct of the study.

\section{References}

1 Parent F, Bachir D, Inamo J, et al. A hemodynamic study of pulmonary hypertension in sickle cell disease. $N$ Engl $J$ Med 2011; 365: 44-53.

2 Fonseca GHH, Souza R, Salemi VMC, et al. Pulmonary hypertension diagnosed by right heart catheterisation in sickle cell disease. Eur Respir J 2012; 39: 112-118.

3 Gordeuk VR, Castro OL, Machado RF. Pathophysiology and treatment of pulmonary hypertension in sickle cell disease. Blood 2016; 127: 820-828.

4 Reiter CD, Wang X, Tanus-Santos JE, et al. Cell-free hemoglobin limits nitric oxide bioavailability in sickle-cell disease. Nat Med 2002; 8: 1383-1389.

5 Morris CR, Kato GJ, Poljakovic M, et al. Dysregulated arginine metabolism, hemolysis-associated pulmonary hypertension, and mortality in sickle cell disease. JAMA 2005; 294: 81-90.

6 Gladwin MT, Vichinsky E. Pulmonary complications of sickle cell disease. N Engl J Med 2008; 359: 2254-2265.

7 Adedeji MO, Cespedes J, Allen K, et al. Pulmonary thrombotic arteriopathy in patients with sickle cell disease. Arch Pathol Lab Med 2001; 125: 1436-1441.

8 Anthi A, Machado RF, Jison ML, et al. Hemodynamic and functional assessment of patients with sickle cell disease and pulmonary hypertension. Am J Respir Crit Care Med 2007; 175: 1272-1279.

9 Mehari A, Igbineweka N, Allen $\mathrm{D}$, et al. Abnormal ventilation-perfusion scan is associated with pulmonary hypertension in sickle cell adults. J Nucl Med 2018; in press [https://doi.org/10.2967/jnumed.118.211466].

10 Mehari A, Alam S, Tian X, et al. Hemodynamic predictors of mortality in adults with sickle cell disease. Am J Respir Crit Care Med 2013; 187: 840-847.

11 Gladwin MT, Sachdev V, Jison ML, et al. Pulmonary hypertension as a risk factor for death in patients with sickle cell disease. N Engl J Med 2004; 350: 886-895.

12 Klings ES, Machado RF, Barst RJ, et al. An official American Thoracic Society clinical practice guideline: diagnosis, risk stratification, and management of pulmonary hypertension of sickle cell disease. Am J Respir Crit Care Med 2014; 189: 727-740.

13 Barst RJ, Mubarak KK, Machado RF, et al. Exercise capacity and haemodynamics in patients with sickle cell disease with pulmonary hypertension treated with bosentan: results of the ASSET studies. Br J Haematol 2010; 149: 426-435.

14 Machado RF, Barst RJ, Yovetich NA, et al. Hospitalization for pain in patients with sickle cell disease treated with sildenafil for elevated TRV and low exercise capacity. Blood 2011; 118: 855-864.

15 Davis BA, Allard S, Qureshi A, et al. Guidelines on red cell transfusion in sickle cell disease. Part II: indications for transfusion. Br J Haematol 2017; 176: 192-209.

16 Tsitsikas DA, Seligman H, Sirigireddy B, et al. Regular automated red cell exchange transfusion in the management of pulmonary hypertension in sickle cell disease. Br J Haematol 2014; 167: 707-710.

17 Galiè N, Humbert M, Vachiery J-L, et al. 2015 ESC/ERS Guidelines for the diagnosis and treatment of pulmonary hypertension. The Joint Task Force for the Diagnosis and Treatment of Pulmonary Hypertension of the European Society of Cardiology (ESC) and the European Respiratory Society (ERS). Eur Respir J 2015; 46: 903-975.

18 Narbey D, Habibi A, Chadebech P, et al. Incidence and predictive score for delayed hemolytic transfusion reaction in adult patients with sickle cell disease. Am J Hematol 2017; 92: 1340-1348.

19 Michot J-M, Driss F, Guitton C, et al. Immunohematologic tolerance of chronic transfusion exchanges with erythrocytapheresis in sickle cell disease. Transfusion 2015; 55: 357-363.

20 Adams RJ, McKie VC, Hsu L, et al. Prevention of a first stroke by transfusions in children with sickle cell anemia and abnormal results on transcranial Doppler ultrasonography. N Engl J Med 1998; 339: 5-11.

21 Lee MT, Piomelli S, Granger S, et al. Stroke Prevention Trial in Sickle Cell Anemia (STOP): extended follow-up and final results. Blood 2006; 108: 847-852.

22 Yung GL, Channick RN, Fedullo PF, et al. Successful pulmonary thromboendarterectomy in two patients with sickle cell disease. Am J Respir Crit Care Med 1998; 157: 1690-1693.

23 Freeman AT, Ataga KI. Pulmonary endarterectomy as treatment for chronic thromboembolic pulmonary hypertension in sickle cell disease. Am J Hematol 2015; 90: E223-E224.

24 Hsieh MM, Fitzhugh CD, Weitzel RP, et al. Nonmyeloablative HLA-matched sibling allogeneic hematopoietic stem cell transplantation for severe sickle cell phenotype. JAMA 2014; 312: 48-56.

25 Pittman C, Hsieh MM, Coles W, et al. Reversal of pre-capillary pulmonary hypertension in a patient with sickle cell anemia who underwent haploidentical peripheral blood stem cell transplantation. Bone Marrow Transplant 2017; 52: 641-642.

26 Delville M, Manceau S, Ait Abdallah N, et al. Arterio-venous fistula for automated red blood cells exchange in patients with sickle cell disease: complications and outcomes. Am J Hematol 2017; 92: 136-140.

27 Olnes M, Chi A, Haney C, et al. Improvement in hemolysis and pulmonary arterial systolic pressure in adult patients with sickle cell disease during treatment with hydroxyurea. Am J Hematol 2009; 84: 530-532. 